\title{
Promoting diagnostics as a global good
}

\author{
The COVID-19 pandemic has reasserted the central role of effective diagnostics in the response to outbreaks. But a \\ lack of coordination still hampers widespread access to these critical tools. A diagnostics agenda for global health \\ is urgently needed for the promotion of diagnostics as a global good and to ensure their delivery.
}

\section{Catharina Boehme, Emma Hannay and Madhukar Pai}

B ecause of the COVID-19 pandemic, diagnostic testing is higher on the political agenda than ever before. This historically neglected area of the health system has been elevated to national and international prominence, with world leaders being briefed on testing rates, and new testing innovations making front-page news. This political attention, although welcome, has not necessarily translated into action, with testing rates still a bottleneck in the response to COVID-19 in many countries, and investments in diagnostic development and scale-up being a fraction of what is needed ${ }^{1}$.

The impact of politics on diagnostic delivery has not all been positive.

Convoluted supply chains have been further strained by border closures, a breakdown in global solidarity, and the emergence of 'diagnostic nationalism' that is favoring countries that are home to diagnostics companies at the expense of others. This matters because there are many low- and middle-income countries with limited or no diagnostics-manufacturing capability. For example, millions of rapid tests for malaria are used across Africa, but none are manufactured on the continent.

There is also growing concern that diagnostics companies might focus exclusively on COVID-19 diagnostics due to the huge demand and greater profits, at the cost of essential tests for tuberculosis, human immunodeficiency virus (HIV), malaria and other critical health needs ${ }^{2}$. Until vaccination is widespread, effective 'test, trace and isolate' interventions, coupled with non-pharmaceutical public-health interventions (e.g., mask wearing and physical distancing), remain the most effective strategy for containing this pandemic.

\section{Keeping diagnostics on the agenda} Although the World Health Organization (WHO) has published a model essential diagnostics list (EDL) ${ }^{3}$ since 2018, surveys in multiple countries have shown limited availability of these essential tests at the primary-care level ${ }^{4-6}$. Systemic lack of investment in testing means basic diagnostic capacity is available in only $1 \%$ of primary-care clinics in many low- and middle-income countries ${ }^{7}$. If these countries do not have the tools available to diagnose common causes of fever or cough, how can they spot potential outbreaks?

Lack of easy access to diagnostic testing, in fact, has made the diagnostic step one of the weakest links in the cascade of care for many common conditions, including tuberculosis, AIDS, diabetes and hypertension ${ }^{8}$. Evidence suggests that this could have a disproportionately negative impact on women, who routinely face greater barriers to accessing high-quality healthcare ${ }^{9}$.

There is an unprecedented opportunity to build back better - to ensure pandemic preparedness, protect the gains made in combating other diseases, and improve global health diagnostics to accelerate progress toward universal health coverage (UHC). To this end, we suggest five priorities (Fig. 1) for the international community for translating the political attention on COVID-19 testing into action for global health.

First, a diagnostics agenda for global health is needed to sustain political attention even after the pandemic subsides. This agenda should identify priorities in research, manufacturing and access to essential diagnostic tools to support pandemic preparedness and UHC. The agenda should be given teeth through declarations and vehicles such as the G20 summit, regional organizations or a World Health Assembly resolution with clear targets and milestones, around which ministers of health and the global health community could rally. We have seen through other important global health diseases, such as AIDS and vaccine-preventable diseases, that gaining visibility in the global political agenda can translate into sustained country and donor attention. The creation of structures such as The Global Fund, or Gavi, the Vaccine Alliance, has supported gains in global health. At present, no equivalent alliance or structure exists to support global health diagnostics.

\section{Five priorities for translating the political attention on} COVID-19 testing into action for global health

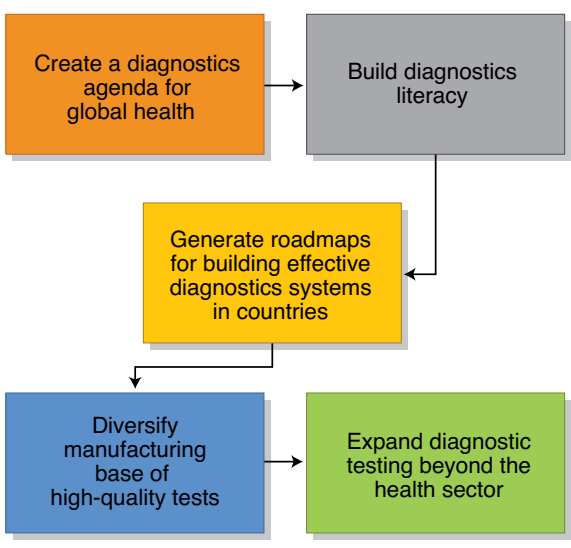

Fig. 1 | Learning from COVID-19 to improve global health diagnostics. Five priorities for promoting diagnostics as a global good.

Second, to help communicate the agenda, building diagnostics literacy among the media, policy makers, health workers and the public must continue. It has been known for a long time that issues of patient and clinician confidence in diagnostic tests have a strong role in how effectively tests are used and how test results are acted upon. The diagnostics community has typically engaged with its own technical experts but does not have a strong track record of explaining key medical and epidemiological concepts in a digestible format for a broader audience. The work of science communicators during the COVID-19 pandemic must be built upon to enhance broader diagnostics literacy both within the health system and in the wider public discourse. This human angle needs to be brought into discussions on the role of diagnostic testing.

Third, to 'action' the global agenda, implementers, researchers and clinicians need to work with political and health-system leadership to create roadmaps for building effective diagnostics systems in countries. Pandemic preparedness might be the immediate trigger for such 
roadmaps in the short term, but over the longer term, the goal should be to create an effective diagnostics response to support UHC, including adequate budgetary provisions. This will require a supportive policy environment, including the creation of national EDLs to establish a package of diagnostic tests to which all patients should have access. Progress thus far has been slow. Although the WHO model EDL was published two years ago, only India has so far formalized its own national EDL.

Fourth, well-conceived country testing strategies may still fail if there is not a diverse manufacturing base for high-quality tests. Currently, the world is critically dependent on a small number of high-income countries that drive innovation and manufacture diagnostic tests. In fact, the first two game-changing rapid diagnostic tests of COVID-19 antigens to receive WHO Emergency Use Listing are manufactured not only in the same country - South Korea - but also in the same neighborhood. This 'trickle-down' model, in which products and innovations are developed in the global north and, after a decade or two, slowly trickle down to the global south, where the biggest needs are, and where technologies often have the greatest impact, must be reconsidered ${ }^{10}$. Manufacturing needs to be focused on resilience as well as efficiency, through diversification in the countries and industries that create innovation in diagnostics and provide the manufacturing base. This may require careful stewardship from countries and regional economic groups, including the creation of supportive regulatory environments to facilitate new entrants.

Fifth, this agenda will be hard to realize if it remains a health-sector issue only. Diagnostic testing must be expanded beyond the health sector to being an issue that affects many areas of society. COVID19 has shown that testing is now everyone's business, with border crossings, schools, workplaces and social gatherings now all needing their own testing strategies, in addition to the use of testing in animal healthcare, wildlife and environmental surveillance. This has implications for the health community, which must learn to engage and partner with a new set of stakeholders with differing norms and technical experience. It does, however, also provide an opportunity for engagement with possible funders beyond the health sector to carry forward access to testing. Access to tests should be democratized and decentralized, for maximal impact. Home-based sample collection and self-testing approaches, combined with tele-health or remote care, might be the dominant approach in future.

\section{Pitfalls of politicization}

To support these five priorities in agenda setting, diagnostics literacy, country roadmaps, manufacturing diversity and testing expansion, the role of international cooperation and global solidarity in providing access for all must be recognized and reinforced, and diagnostics must begin to be promoted as a global good. Access to testing cannot become a political target to trade or a target for nationalism to block access for others. And access to testing should not be seen as a luxury or privilege. Early in the COVID-19 crisis, countries blocked the exportation of raw ingredients and consequently deprived themselves of the finished diagnostic tests they needed to respond to the pandemic. Altering the global narrative and building resilience into the system will only go so far if the negative impact of politics on testing is not managed too.

\section{Pivoting to 'build back better'}

The pressing need for expanded diagnostic testing in COVID-19 is not over, and many countries are still grappling with surging demand in the face of substantial access issues. While researchers and clinicians work to solve this acute crisis, there is a need to also pivot and plan to build back better with a new global agenda for diagnostic testing that can support both pandemic preparedness and UHC. To not learn the lessons from COVID-19 diagnostic testing would be a terrible waste. The right kind of political attention for testing must be sustained and converted into action for better access to diagnostics.

\section{Catharina Boehme', Emma Hannay ${ }^{1}$ and} Madhukar Pai (D) 2ه

${ }^{1}$ FIND, Geneva, Switzerland. ${ }^{2}$ School of Population and Global Health, McGill University, Montreal, Canada.

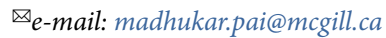

Published online: 15 March 2021

https://doi.org/10.1038/s41591-020-01215-3

References

1. World Health Organization. https://www.who.int/publications/i/ item/an-economic-investment-case-financing-requirements (2020).

2. Venkatesan, P. Lancet Microbe 1, e64 (2020).

3. World Health Organization. https://www.who.int/medical_ devices/publications/Standalone_document_v8.pdf?ua=1 (2019).

4. Kohli, M. et al. Lancet Infect. Dis. 18, 1064-1065 (2018).

5. Sánchez-Sánchez, J. et al. Microbes Infect. https://doi. org/10.1016/j.micinf.2020.09.007 (2020).

6. Shumbej, T. et al. Trop. Dis. Travel Med. Vaccines 6, 4 (2020).

7. Leslie, H. H., Spiegelman, D., Zhou, X. \& Kruk, M. E. Bull. World Health Organ. 95, 738-748 (2017).

8. Pai, M., Walia, K. \& Boehme, C. C. Lancet Public Health 4 , e492 (2019).

9. Women in Global Health \& FIND. https://www.womeningh.org/ testing (2020).

10. Pai, M. Forbes https://www.forbes.com/sites/madhukarpai/ 2020/02/17/global-health-technologies-time-to-re-thinkthe-trickle-down-model/?sh=236244b544d9 (17 February 2020).

Competing interests

C.B. and E.H. are currently employed by FIND, and M.P. serves as an advisor to FIND. FIND is a not-for-profit foundation that supports the development, evaluation and implementation of several diagnostics, including COVID-19. FIND has product evaluation agreements with several private sector companies that design diagnostics for global health. These agreements strictly define FIND's independence and neutrality vis-a-vis the companies whose products get evaluated and describe roles and responsibilities. FIND is co-convener of the Access to COVID-19 Tools (ACT) Accelerator, a partnership to accelerate access to the diagnostics tools needed in the COVID-19 response. 